\title{
Study of Low Temperature Curable Polybenzoxazole Precursors Containing Cyclohexanedicarboxylic Acid Structures
}

\author{
Ken-ichi IWASHITA, Takashi HATTORI, and Tomonori MINEGISHI \\ Research \& Development Center, Hitachi Chemical Co. Ltd. \\ 4-13-1 Higashi, Hitachi, Ibaraki, 317-855, Japan \\ k-iwashita@hitachi-chem.co.jp
}

\begin{abstract}
Keywords: polybenzoxazole, low temperature curing, cyclohexanedicarboxylic acid,
\end{abstract} configurational isomer, photolithography

\section{Introduction}

Photodefinable polybenzoxazoles (PD-PBOs) have been applied to insulating and buffer coatings for semiconductor devices in silicon technology, because of their excellent electrical, thermal, and mechanical properties afforded by high thermal treatment at over $300^{\circ} \mathrm{C}$ [1]. However, it has been reported that low temperature curable materials are required to obtain successful result in such applications as the gate dielectric layers and insulation layers for novel organic devices $[2,3]$.

We have already reported that the poly(o-hydroxyamide)s (PHAs) containing alicyclic structures as $\mathrm{PBO}$ precursors showed good film properties with lower curing temperatures [4]. Cyclohexanedicarboxylic acid (CHDA) as one of the alicyclic monomers has two isomers of cis- and trans-configurations. In this paper, we investigated the cyclization percentage and the photolithographic properties of the PHAs containing CHDA structures for low temperature curing.

\section{Method}

PHAs were prepared from three kinds of dicarboxylic acids and a bis (o-aminophenol) by an acid chlorination method (Figure 1). Both the photoactive compound diazonaphtoquinone (DNQ) and the solvent N-methyl-2-pyrrolidone (NMP) were used as purchased.

A solution of the PHA was spin-coated on a silicon wafer, which was then soft-baked on a hot plate at $120^{\circ} \mathrm{C}$ for $3 \mathrm{~min}$ to give a thin film whose thickness was adjusted to $15 \mu \mathrm{m}$. The resulting film was cured at $200^{\circ} \mathrm{C}$ for $1 \mathrm{~h}$ under a nitrogen atmosphere to measure the cyclization percentage by infrared (IR) spectroscopy (DIGILAB FTS-3000). Differential scanning calorimetry (DSC) (SII DSC-6200) was used to monitor the cyclization of PHAs.

A photopolymer solution was prepared by dissolving PHA, DNQ, and other additives such as adhesion promoter in NMP. The solution was spin-coated on a silicon wafer, soft-baked on a hot plate, and exposed to the radiation of a contact aligner and an $i$-line stepper (Canon FPA-3000iw). The puddle method with $2.38 \%$ aqueous TMAH as the developer was applied for the development.

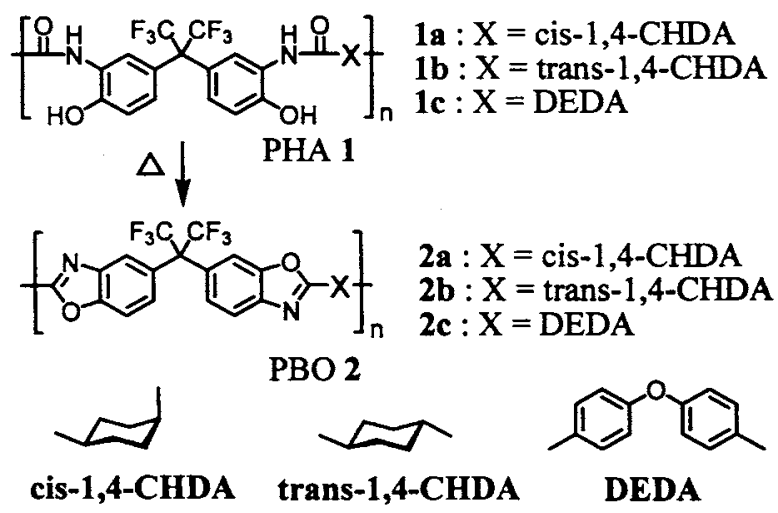

Figure 1. Structures of polymers studied.

\section{Results and Discussion}

\subsection{Thermal cyclization behavior}

To observe the effect of PHA structures on thermal cyclizaton, we synthesized several PHA 1s that were derived from cis- and trans-1,4-CHDAs and aromatic dicarboxylic acid. The molecular 
weights of 1a, 1b, and $1 \mathrm{c}$ were $33,600,51,000$ and 24,000 , respectively. Table 1 shows the cyclization percentage of PHA 1s estimated from the IR spectra of the PHA films cured at $200^{\circ} \mathrm{C}$ for $1 \mathrm{~h}$ on silicon wafers. We found that PHA 1a and 1b containing CHDA structures showed higher cyclization percentage than the aromatic PHA 1c. As for the structure of CHDA, 1a containing cis-configuration showed higher cyclization percentage of $44 \%$.

Next, we investigated the thermal cyclization behavior of the PHA 1s in detail by DSC. As can be seen in Figure 2, PHA 1s exhibited endothermic peaks caused by thermal cyclization from 200 to $350^{\circ} \mathrm{C}$. As a result, $1 \mathrm{a}$ showed the lowest maximum cyclization temperature.

It was reported that $\mathrm{Tg}$ value correlates with the rigidity of polymer chains on poly(ether sulfone)s and polyimides $[5,6]$. We measured the $\mathrm{Tg}$ of PBO 2s derived from $350^{\circ} \mathrm{C}$-cured films of PHA 1s. As shown in Table 2, 2a showed a lower Tg than $\mathbf{2 b}$ and $2 \mathbf{c}$. These results suggest that $2 \mathbf{a}$ has relatively flexible polymer chains, resulting in a higher cyclization percentage. The cis-1,4-CHDA bend structure which leads to less intermolecular interaction between the polymers may also contribute to the flexible structure.

Table 1. Property of PHA 1 films cured at $200^{\circ} \mathrm{C}$.

\begin{tabular}{|c|c|c|c|c|}
\hline Item & Unit & $\mathbf{1 a}$ & $\mathbf{1 b}$ & $\mathbf{1 c}$ \\
\hline Cyclization & $\%$ & 44 & 22 & 14 \\
\hline
\end{tabular}

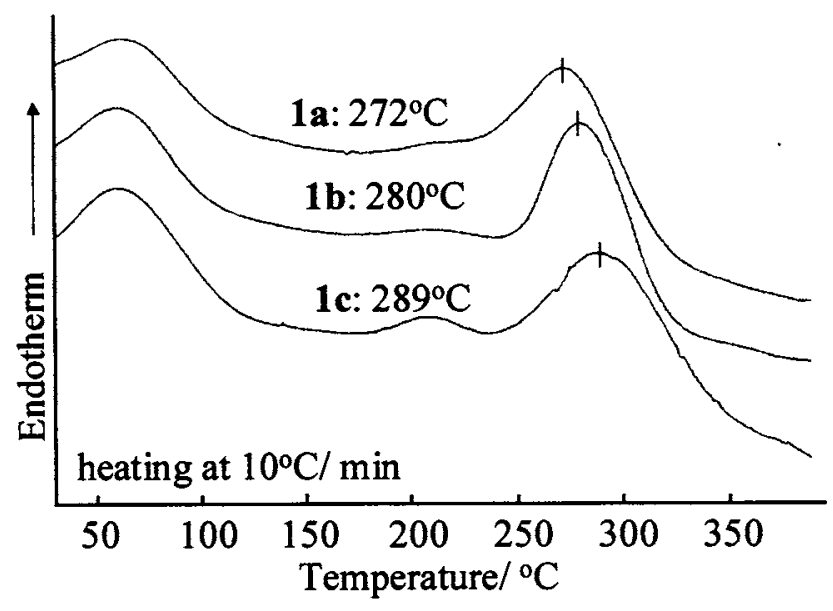

Figure 2. DSC measurements for $1 \mathbf{a}, \mathbf{1 b}$, and $1 \mathbf{c}$.
Table 2. Cured film property of PBO 2s.

\begin{tabular}{|c|c|c|c|c|}
\hline Item & Unit & 2a & 2b & 2c \\
\hline $\mathrm{Tg}$ & ${ }^{\circ} \mathrm{C}$ & 242 & 248 & 266 \\
\hline
\end{tabular}

\subsection{Photolithographic performance}

Photolithographic properties of the PHA containing the promising CHDA structure in DNQ systems was evaluated on a $12-\mu \mathrm{m}$-thick prebaked film. The sensitivity $\left(D_{0}:\right.$ dose to clear) was 200 $\mathrm{mJ} / \mathrm{cm}^{2}$ along with $80 \%$ film retention after $2.38 \%$ aqueous TMAH development. The scanning electron micrograph (SEM) of the obtained photoimage was shown in Figure 3. It was found that the PHA containing CHDA structure could be applied for aqueous developable PD-PBO.

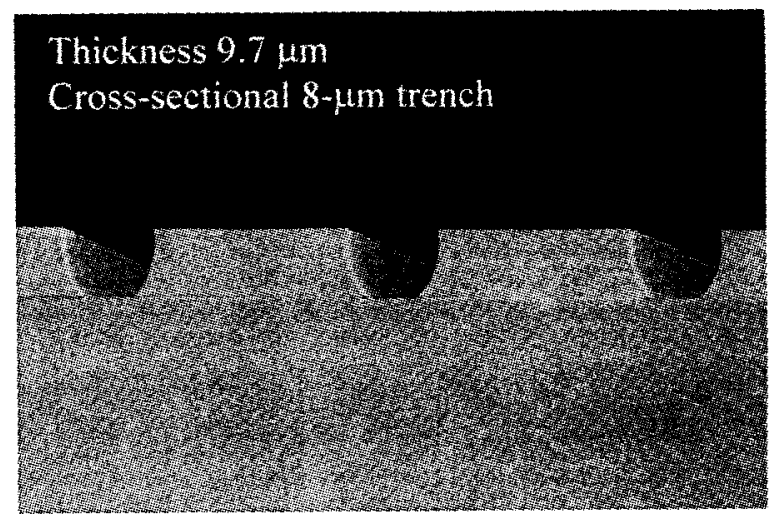

Figure 3. SEM photograph after development.

\section{Conclusion}

We focused on PHAs containing CHDA structures. It was found that the PHA derived from cis-1,4-CHDA showed high cyclization percentage at the curing temperature of $200^{\circ} \mathrm{C}$. In addition, fine resolution of pattern was obtained from the PHA containing CHDA in DNQ system.

\section{References}

[1] R. Rubner, Adv. Mater., 2 (1990) 452.

[2] Y. Kato, S. Iba, R. Teramoto, T. Sekitani, T. Someya, H. Kawaguchi, and T. Sakurai, Appl. Phys. Lett., 84 (2004) 3789.

[3] R. Okada, K. Miyoshi, N. Arai, and M. Tomikawa, J. Photopolym. Sci. Technol., 17 (2004) 207.

[4] K. Iwashtia, T. Hattori, T. Minegishi, S. Ando, F. Toyokawa, and M. Ueda, J. Photopolym. Sci. Technol., 19 (2006) 281.

[5] V. Carlier, J. Devaux, R. Legras, and P. T. McGrail, Macromolecules, 25 (1992) 6646.

[6] H. Matsutani, T. Hattori, M. Ohe, T. Ueno, R. L. Hubbard, and Z. Fathi J. Photopolym. Sci. Technol., 18 (2005) 327. 